\title{
TRANSPORTE INTRA-HOSPITALAR DO PACIENTE CRÍTICO
}

\author{
INTRA-HOSPITAL TRANSPORT OF CRITICAL PATIENTS
}

\author{
Gerson A. Pereira Júnior ${ }^{1}$, Júlia Batista de Carvalho², Arnóbio D. Ponte Filho³, \\ Daniela A. Malzone ${ }^{4}$, César E. Pedersoli ${ }^{5}$
}

\begin{abstract}
${ }^{1}$ Médico Assistente, Unidade de Emergência, Hospital das Clínicas da Faculdade de Medicina de Ribeirão Preto - USP. Docente, Emergências Médicas e Habilidades Cirúrgicas, Curso de Medicina da Universidade de Ribeirão Preto (UNAERP). ${ }^{2}$ Médica, Residente de Medicina Intensiva, UNAERP. ${ }^{3}$ Médico, Residente de Clínica Médica, Hospital do Servidor Público Municipal de São Paulo. ${ }^{4}$ Docente, Ginecologia, UNAERP. ${ }^{5}$ Enfermeiro, Unidade de Emergência, Hospital das Clínicas da Faculdade de Medicina de Ribeirão Preto - USP. Docente, Emergências Médicas e Atendimento Pré-hospitalar, Curso de Medicina - UNAERP.

CorrespondênciA: Dr. Gerson Alves Pereira Júnior. Rua Bernardino de Campos, 1000. Higienópolis, CEP 14030-150 - Ribeirão Preto - SP. gersonapj@gmail.com.br
\end{abstract}

Pereira Júnior GA, Carvalho JB, Ponte Filho AD, Malzone DA, Pedersoli CE. Transporte intra-hospitalar do paciente crítico. Medicina (Ribeirão Preto) 2007; 40 (4): 500-8, out./dez.

RESUMO: O avanço do conhecimento médico e o desenvolvimento da tecnologia para diagnóstico e tratamento das doenças que afligem os seres humanos têm permitido um aumento da longevidade das pessoas hígidas e daquelas que possuem agravos agudos ou crônicos. Embora estes avanços estejam heterogeneamente distribuídos, há uma nítida melhora das condições de atendimento médico em nosso meio e isto tem feito com que pacientes que anteriormente evoluíssem para o óbito, tenham condições de se manterem vivos em diferentes condições de qualidade de vida. Isto tem feito com que os hospitais tenham que aumentar as áreas de atendimento e cuidados de pacientes críticos. Estes pacientes têm sido beneficiados pela tecnologia de diagnóstico, principalmente, de imagem existente e, quase sempre, para ter acesso a estes benefícios, estes pacientes precisam ser transportados para fora da área de cuidados intensivos e manter o mesmo nível de monitorização para que não haja problemas. É aí que está a grande importância do transporte do paciente crítico que, no geral, vem sendo muito negligenciada pelos profissionais de saúde. O objetivo deste artigo é fazer uma reflexão dos vários momentos, fases e cuidados envolvidos no transporte intra-hospitalar, discutindo as suas diversas modalidades.

Descritores: Transporte de Pacientes. Cuidados Intensivos. Monitoramento. Transferência de Pacientes.

\section{1- INTRODUÇÃO}

A decisão de transportar um paciente crítico deve ser baseada na avaliação e ponderação dos benefícios e riscos potenciais. A razão básica para o transporte do paciente crítico é a necessidade de cuidados adicionais (tecnologia e/ou especialistas) não disponíveis no local onde o paciente se encontra ${ }^{1}$.

$\mathrm{O}$ transporte pode ser intra ou inter-hospitalar. O transporte intra-hospitalar é necessário para a realização de testes diagnósticos (tomografia computa- dorizada, ressonância nuclear magnética, angiografias, dentre outros para intervenções terapêuticas (como para o centro cirúrgico) ou para a internação em centro de terapia intensiva (CTI). O transporte inter-hospitalar é realizado sempre que se necessita de maiores recursos humanos, diagnósticos, terapêuticos e de suporte avançado de vida, que não estão presentes no hospital de origem.

O transporte do paciente crítico sempre envolve uma série de riscos, sendo que o problema mais freqüente é a falha no controle das funções cardior- 
respiratórias, resultando em instabilidade fisiológica com prejuízo da oxigenação tecidual, que pode trazer sérias consequiências ${ }^{2}$. Podem ainda ocorrer outras alterações, tais como hipertensão grave, arritmias, obstrução aérea, entre várias outras ${ }^{2}$.

Alguns riscos são inerentes ao transporte, independente do tempo ou distância a ser percorrida ${ }^{1,2,3}$. As causas dessas alterações não são sempre facilmente explicadas, até porque elas podem não ser detectadas sem monitorização adequada. Tais alterações fisiológicas não estão sempre relacionadas a erros técnicos e podem ser devidas a alterações respiratórias e cardíacas resultantes da dor provocada pelo movimento do paciente no seu deslocamento, ou alterações decorrentes da mudança de decúbito do paciente. $\mathrm{Ou}-$ tras, no entanto, são decorrentes de falhas técnicas como a interrupção acidental da infusão endovenosa de aminas vasoativas, a perda de pressão nos cilindros de oxigênio e outras explicações plausíveis, porém geralmente não documentadas ${ }^{3 / 6}$.

Pelo fato do período de transporte ser de potencial instabilidade, deve sempre ser questionado se os testes diagnósticos ou as intervenções terapêuticas alterarão o tratamento e o prognóstico do paciente ${ }^{1,3,4}$. Isto nem sempre é bem avaliado. Trabalhos clínicos demonstram uma mudança na conduta terapêutica em apenas 29 a $39 \%$ dos pacientes após exames diagnósticos, enquanto $68 \%$ deles tiveram sérias alterações fisiológicas durante o transporte ${ }^{4,7 / 10}$.

$\mathrm{O}$ risco ao paciente durante o transporte pode ser minimizado por meio de um planejamento cuidadoso, qualificação do pessoal responsável pelo transporte e seleção de equipamentos adequados ${ }^{1,4}$. Não deve haver nenhum momento no transporte sem monitorização ou manutenção das funções vitais do paciente. Os equipamentos disponíveis e o nível de prática dos recursos humanos devem ser iguais às intervenções requeridas ou antecipadas para o paciente ${ }^{1}$. Dependendo das características de cada hospital, podese optar pela presença de uma equipe de transporte especializada, tanto para o transporte intra, quanto inter-hospitalar ${ }^{4,5}$.

A predição de risco para um grupo específico de pacientes não é bem determinada, ou seja, não se sabe qual o grupo de pacientes que terá maior ou menor chance de deterioração fisiológica durante o transporte. Contudo, o grupo de pacientes com maiores chances de deterioração do quadro clínico durante o transporte parece ser daqueles - com falência respiratória e baixa complacência pulmonar, identificados por necessitarem de elevada pressão positiva no final da expiração (PEEP) durante a ventilação mecânica ${ }^{4,7}$.

Embora muitos serviços ainda utilizem a ventilação manual (AMBU), estudos recentes mostram que a utilização de ventiladores de transporte com pressão de suporte são capazes de manter uma ventilação mais consistente, quando se analisa a relação $\mathrm{PaO}_{2} / \mathrm{FiO}_{2}$, comparando-se pacientes ventilados manualmente e com ventiladores mecânicos ${ }^{11}$. A ventilação manual resulta em colapso alveolar, diminuição da complacência, hipoventilação e diminuição da saturação arterial de oxigênio. Os ventiladores portáteis para transporte mantêm um nível maior e constante de PEEP, porém são de alto custo ${ }^{2}$. Por outro lado, para outros grupos de pacientes transportados, analisados por tipo de doença, idade, transporte para cirurgia, pressão arterial de oxigênio inicial ou tempo de transporte não registraram diferenças significativas entre as duas modalidades de ventilação ${ }^{2,10}$.

Apesar de opiniões divergentes quanto à estratificação dos pacientes por gravidade ${ }^{12 / 16}$, é freqüente considerarmos como mais graves e de maior risco no transporte, os pacientes com necessidade de PEEP > $14 \mathrm{~cm} \mathrm{H}_{2} \mathrm{O}$ e aqueles em uso de drogas vasoativas, como dobutamina, dopamina e noradrenalina ${ }^{17}$.

Existe também a possibilidade de eventos de menor relevância, embora também potencialmente fatais e cuja fácil previsibilidade torna inadmissível sua ocorrência. Os principais incidentes verificados em estudos clínicos foram: desconexão da leitura do eletrocardiograma, falha do monitor, infiltração inadvertida do tecido subcutâneo pela perda não percebida do acesso venoso e desconexão da infusão de drogas vasoativas e sedação, que ocorreram em $34 \%$ dos transportes ${ }^{4,6,7,8}$. Destes, ao contrário do previsto, a maior parte $(60 \%)$ ocorreu nos transportes eletivos e a menor parte $(40 \%)$ ocorreu nos transportes de emergência; o que pode refletir uma maior preparação e monitorização nas situações emergenciais e maior displicência nas situações eletivas ${ }^{8}$.

O principal fator determinante da qualidade dos cuidados durante o transporte é o treinamento e a eficiência da equipe de transporte ${ }^{1,2}$. Os equipamentos para monitorização são, obviamente, importantes e a sua miniaturização tem resolvido muitos dos problemas associados com a falta de espaço, sendo também menos susceptíveis a artefatos de movimento. Além disso, o desenvolvimento de métodos diagnósticos portáteis, como é o caso do ultrassom, doppler transcraniano e eletroencefalografia, poderá diminuir a necessidade de transporte dos pacientes críticos ${ }^{2}$. 
Existem 4 principais tipos de transferência intrahospitalar e há considerações importantes acerca de cada uma delas:

a) Transferência, sem retorno do paciente, para fora da área de tratamento intensivo (CTI, centro cirúrgico e sala de recuperação anestésica)

Envolve a transferência dos pacientes com alta médica da sala de recuperação anestésica ou do CTI. Espera-se que sejam pacientes com maior estabilidade hemodinâmica, sendo esta a transferência menos perigosa dentre os transportes intra-hospitalares.

O principal cuidado é evitar a hipóxia, mantendo a via aérea permeável (evitar obstrução por secreções ou queda do nível de consciência), evitar uma diminuição da capacidade ventilatória (pela dor ou queda do nível de consciência) e fornecer oxigênio suplementar, se necessário. Normalmente, não é necessária a presença de médico neste tipo de transporte.

\section{b) Transferência em um único sentido de um pa- ciente para uma área de cuidados intensivos}

Envolve o transporte de pacientes da sala de emergência (clínica ou de trauma) ou enfermaria para o CTI ou para o centro cirúrgico. Deve ser realizado, idealmente, após reposição volêmica e estabilização do paciente.

Os cuidados são uma extensão do atendimento inicial: evitar hipóxia (preferencialmente, usar o oxímetro de pulso mantendo saturação maior que $94 \%$ ), evitar deslocamentos de tubos endotraqueais, sondas vesicais e nasogástricas, drenos de tórax e cateteres intravenosos.

Deve-se estar sempre atento aos parâmetros hemodinâmicos e corrigir as alterações dentro do possível, sendo obrigatória a presença de médicos neste tipo de transporte.

\section{c) Transferência do CTI para o centro cirúrgico, com retor- no ao CTI}

A necessidade de intervenções cirúrgicas em qualquer segmento do corpo torna necessário o transporte do paciente crítico, man- tendo o mesmo nível de cuidados no trajeto e dentro do centro cirúrgico. Tais procedimentos devem ter uma indicação precisa e em tempo, num acordo entre o cirurgião, o anestesista e o intensivista.

Alguns procedimentos cirúrgicos podem ser realizados à beira do leito, dentro do CTI, evitando-se o transporte. Dentre esses procedimentos cirúrgicos estão a traqueostomia, gastrostomia percutânea, inserção de filtros de veia cava inferior, a ventriculostomia, relaparotomias programadas para peritonite grave com o abdome aberto ou abertura da parede abdominal em pacientes com síndrome compartimental abdominal.

\section{d) Transferência do CTI para áreas não-CTI e retorno do paciente ao CTI}

Envolve as transferências para procedimentos diagnósticos ou terapêuticos não cirúrgicos, principalmente para o setor de radiologia. Incluem-se aí também os pacientes transportados da sala de emergência para os estudos diagnósticos, pois estes pacientes também saem da área de cuidados mais intensivos (Figura 1).

A tecnologia e a sofisticação dos métodos diagnósticos não portáteis que auxiliam os médicos no diagnóstico de problemas complexos requerem o transporte

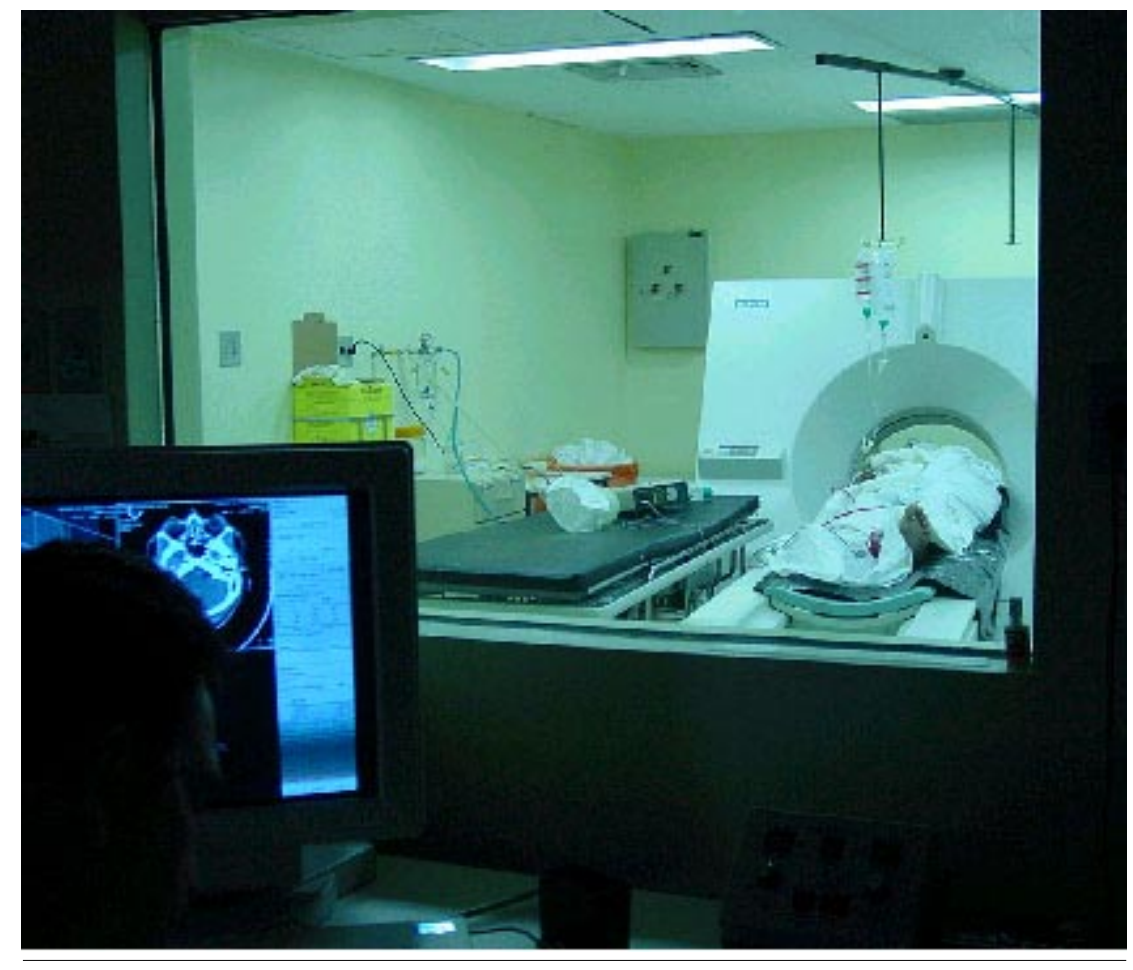

Figura 1. Acompanhamento e monitorização de paciente traumatizado durante a realização da tomografia computadorizada de crânio. 
de pacientes críticos para fora do ambiente protegido da CTI por períodos de tempo que podem ser prolongados.

A maior parte dos exames diagnósticos compreende a realização de exames de tomografia computadorizada do abdome para detectar pancreatite necro-hemorrágica ou abscessos intra-abdominais, do tórax para excluir abscesso pulmonar ou empiema, do crânio para seguimento do trauma crânio-encefálico e acidentes vasculares cerebrais, punções percutâneas guiadas por tomografia e angiografia para detecção de complicações tromboembólicas ou confirmação do diagnóstico de morte encefálica ${ }^{8,9,10}$.

Os exames de imagem devem ser prontamente avaliados após a sua realização devido a possível necessidade de repetição, poupando desta forma um novo transporte.

Atualmente, com o avanço da tecnologia, alguns métodos de diagnóstico por imagem podem ser realizados à beira do leito, eliminando o risco inerente ao transporte. Assim, o ultrassom (tórax, abdome e de membros), doppler transcraniano, ecocardiograma transtorácico e transesofágico e, mais recentemente, a tomografia computadorizada portátil têm sido aperfeiçoados para melhor definição das imagens e segurança para demais pacientes e equipe ${ }^{18,19}$.

Os passos a serem seguidos no transporte intrahospitalar do paciente crítico envolve 3 fases: ${ }^{20,21,22}$

1 Fase preparatória

2. Fase de transferência

3. Fase de estabilização pós-transporte

\section{1- FASE PREPARATÓRIA:}

\section{1- Coordenação e comunicação pré-transporte}

Inicialmente, deve-se avaliar a gravidade e a condição atual do paciente, promovendo a melhor estabilização cardiorrespiratória possível.

Deve certificar-se de que o local de destino esteja aguardando o paciente, estimando o tempo de transporte e averiguando se os equipamentos de monitorização, rede de gases, ventiladores mecânicos e de aspiração do local estejam funcionantes. Caso o local de destino seja em andar diferente, o elevador deve estar pronto para a chegada do paciente.

Se o paciente for transportado por outra equipe que não a que assiste o paciente, deve-se informar sobre suas condições gerais, tratamento instituído pré-transporte e estimar suas necessidades pós-trans- porte (tipo e modo ventilação, uso de drogas, dentre outros).

A documentação no prontuário médico deve incluir as indicações para o transporte e o estado do paciente.

\section{2- Equipe de transporte}

O número de pessoas que participarão do transporte é variável, de acordo com a gravidade e complexidade da situação clínica do paciente e do número de equipamentos exigidos. Um número mínimo de duas pessoas é necessário, para ser capaz de providenciar suporte de vias aéreas, interpretar possíveis alterações cardiovasculares e respiratórias e que estejam aptos a lidar com possíveis problemas técnicos nos vários equipamentos.

Podem fazer parte da equipe de transporte: enfermeiro, médico, auxiliares e técnicos de enfermagem e fisioterapeuta.

O médico deve acompanhar o transporte intrahospitalar daqueles pacientes com o estado fisiológico instável e que podem precisar de intervenções agudas que estão além da capacidade técnica do enfermeiro e/ou fisioterapeuta, sendo obrigatória a sua presença nas seguintes situações:

- pacientes com via aérea artificial (intubação endotraqueal, crico/traqueostomia);

- presença de instabilidade hemodinâmica;

- uso de drogas vasoativas;

- utilização de monitorização invasiva, tais como o uso de cateter de artéria pulmonar (Swan-Ganz), pressão intracraniana (PIC), pressão arterial invasiva e cateter no bulbo jugular.

Alguns serviços médicos possuem uma equipe de transporte intra e inter-hospitalar fixa, enquanto outros têm suas equipes formadas a partir de médicos, enfermeiras e técnicos de enfermagem da escala de trabalho no período que vai ocorrer o transporte. Algumas instituições médicas possuem protocolos de transporte intra-hospitalar que incluem o médico em todos os tipos de transporte ${ }^{19,20}$.

\section{3- Equipamentos necessários para o trans- porte}

É fundamental avaliar a necessidade individual dos equipamentos para o transporte de cada paciente, a fim de evitar a sua ausência ou falta de funcionamento longe do local de origem, onde os mesmos podem não estar disponíveis. 
A seguir, são comentados os cuidados principais a serem tomados em vários itens durante o transporte.

- Tubo endotraqueal: assegurar que a fixação esteja adequada. A cânula deve ser aspirada de maneira vigorosa previamente ao transporte e assegurar que seja possível realizar novas aspirações no local de destino. Em locais que não dispõe de aspiração na rede de vácuo hospitalar, pode ser necessário o uso de um aspirador portátil.

\section{- Dispositivos de ventilação:} preferencialmente, devem-se utilizar ventiladores mecânicos portáteis que possuam modos ventilatórios controlados e de suporte, tanto em pacientes sedados, quanto em pacientes conscientes (Figura 2).

$\mathrm{O}$ uso de AMBU conectado ao cilindro de oxigênio é outra opção de ventilação. Os cilindros de oxigênio podem estar acoplados à maca de transporte ou serem transportados em carrinho manual. A desvantagem dos carrinhos manuais com cilindros de oxigênio é a necessidade de uma pessoa a mais no transporte e a maior dificuldade de seu manuseio. Deve ser usado um AMBU de volume adequado ao peso do paciente e a

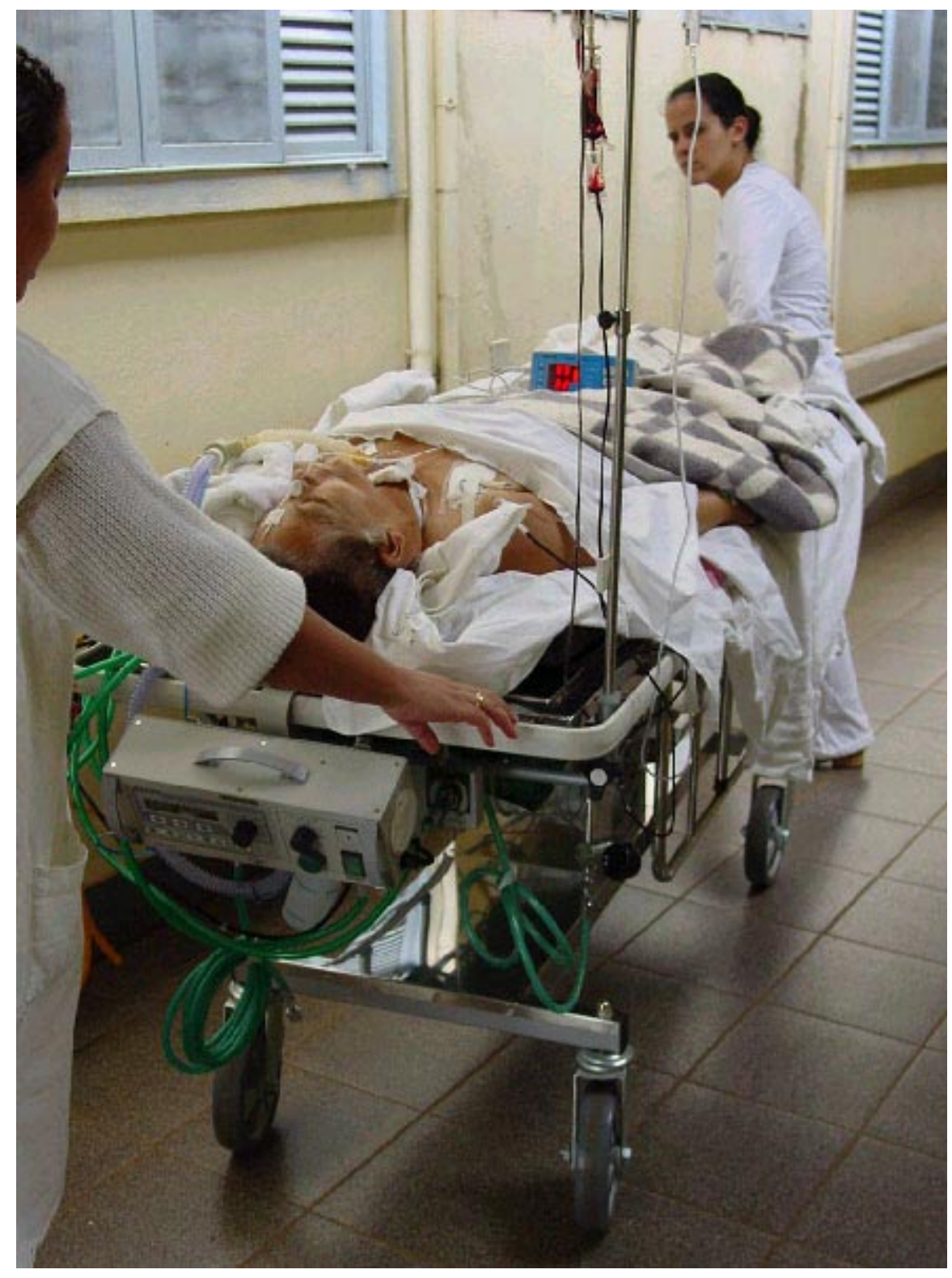

Figura 2. Fase de transferência de paciente dentro do hospital, utilizando um ventilador de transporte. freqüência de ventilação manual deve ser igual a do ventilador previamente ao transporte. $\mathrm{O}$ volume de ar a ser insuflado deve ser adequado para a expansão bilateral e simétrica da parede torácica do paciente $(8$ a $10 \mathrm{ml} / \mathrm{Kg})$, mantendo uma relação inspiração: expiração em torno de 1:2 a 1:323. Deve-se utilizar um AMBU com reservatório, capaz de fornecer $\mathrm{FiO}_{2}$ de 85 a $100 \%$ e, de preferência, com dispositivos capazes de ajustes de PEEP de até $15 \mathrm{~cm}$ $\mathrm{H}_{2} \mathrm{O}$ e de monitorização da pressão intra-traqueal ${ }^{8,23}$.

Nos pacientes conscientes, deve-se tentar a sincronização do AMBU com os movimentos ventilatórios do paciente, no entanto, mesmo para profissionais experientes há uma tendência à hiperventilação e um maior trabalho respiratório do paciente ${ }^{11}$.

Por razões práticas, em adultos, uma $\mathrm{FiO}_{2}$ de $100 \%$ é mais segura durante o transporte, pois esta abordagem elimina a necessidade de um tanque de ar comprimido e um misturador de oxigênio. Já no transporte neonatal, a $\mathrm{FiO}_{2}$ deve ser precisamente controlada $^{8}$. Uma redução de $20 \%$ ou mais na relação $\mathrm{PO}_{2} / \mathrm{FiO}_{2}$, que mostra uma deterioração grave da função respiratória pode ser observada em até $43 \%$ dos pacientes, sendo que em $20 \%$ destes, esta condição persiste por mais de 24 horas $^{2,8}$. A principal variável 
relacionada a esta deterioração é a necessidade de $\operatorname{PEEP}^{2,8,23,24}$.

A medida e o controle acurado do PEEP durante o transporte não é fácil, particularmente quando há alterações na postura do paciente, sedação e alterações do fluxo sangüíneo pulmonar, que podem contribuir para o aumento do "shunt" pulmonar ${ }^{2}$.

Devemos sempre checar a precisão entre o nível de PEEP da escala da válvula (do AMBU ou ventilador mecânico) com a leitura do manômetro (do AMBU ou ventilador mecânico) para determinar o nível correto de PEEP que está sendo utilizada ${ }^{8}$.

$\mathrm{O}$ transporte intra-hospitalar para pacientes com ventilação mecânica e sem níveis elevados de PEEP (menor ou igual a $5 \mathrm{cmH}_{2} \mathrm{O}$ ) é relativamente seguros, sendo transitórias as alterações nas trocas gasosas. Para pacientes em ventilação mecânica com níveis elevados de PEEP, os riscos do transporte devem ser ponderados cuidadosamente, podendo-se lançar mão de procedimentos cirúrgicos à beira do leito e métodos diagnósticos alternativos. Em tais pacientes, o transporte intra-hospitalar deve ser realizado com o uso de ventiladores mecânicos que permitam o mesmo modo ventilatório e níveis elevados de PEEP aplicados no CTI.

Os ventiladores mecânicos portáteis, freqüentemente utilizados, são incapazes de duplicar os padrões de fluxo de gases dos modernos ventiladores microprocessados e não conseguem acuradamente providenciar a ventilação com relação inspiração/ expiração invertida, que gera o PEEP intrínseco ${ }^{2,7,8,24}$. Assim, pode-se lançar mão de unidades móveis de transporte com o mesmo tipo de ventilador mecânico utilizado no $\mathrm{CTI}^{7,8,24}$.

O controle mais rigoroso dos parâmetros ventilatórios é exigido nos pacientes com síndrome da angústia respiratória aguda (SARA), pois a redução acentuada da complacência pulmonar leva ao colapso não homogêneo dos alvéolos, perda da capacidade residual funcional e aumento do "shunt" intrapulmonar, com alterações da relação ventilação/perfusão. Assim, tais pacientes necessitam de ventiladores mecânicos que tenham a possibilidade de promover níveis elevados de PEEP e novos modos de ventilação mecânica, tais como pressão controlada, pressão controlada com inversão da relação inspiração:expiração e volume controlado com pressão regulada, para oxigenação e ventilação mais efetivas ${ }^{24}$. Nestes pacientes com SARA, a súbita desconexão da pressão alveolar positiva ou interrupção da pressão de distensão alveolar efetiva durante a desconexão do ventilador me- cânico pode resultar em diminuição da saturação da hemoglobina e hipoxemia no curso de alguns minutos, o que também ocorre se tentarmos ventilar tais pacientes com AMBU ou ventiladores comuns de transporte ${ }^{24}$.

Deve certificar-se que o cilindro de oxigênio esteja carregado e adotar um fluxo necessário para manter a saturação de oxigênio maior que $94 \%$, registrado no oxímetro de pulso.

- Drogas: uma lista de medicações apropriadas para uso durante o transporte dos diversos tipos de pacientes: pediátricos ${ }^{25,26}$, neonatais ${ }^{27}$, obstétricos ${ }^{28} \mathrm{e}$ adultos $^{29}$, já existe.

Uma caixa de medicações de emergências deve acompanhar o transporte do paciente crítico, sendo que as principais são: adrenalina, amiodarona, lidocaína, atropina, bicarbonato de sódio, adenosina, cloreto de cálcio, dexametasona, dopamina, furosemida, manitol, magnésio, naloxone, nitroglicerina, nitroprussiato de sódio, fenitoína, cloreto de potássio e benzodiazepínicos.

Deve-se antecipar a necessidade de medicações prescritas para o paciente, particularmente, quando o seu uso for intermitente, como é o caso da sedação e bloqueadores neuromusculares.

- Monitor de transporte: existem monitores modulares de transporte que permitem o acompanhamento contínuo de vários parâmetros vitais: eletrocardiografia, oximetria de pulso, capnografia, pressão arterial não invasiva e módulos de pressão invasiva.

$\mathrm{Na}$ ausência de um monitor de múltiplos parâmetros específico para transporte, pelo menos, o oxímetro de pulso deve, obrigatoriamente, acompanhar o transporte de todos os pacientes críticos. Sua bateria deve estar carregada.

- Bombas de infusão: devem ser levadas no transporte de pacientes que necessitem de controle rigoroso da infusão de medicações, tais como: aminas vasoativas, sedativos, bloqueadores neuromusculares, heparina, dentre outros. Fixá-las adequadamente e certificar-se de que as baterias estejam carregadas.

- Dreno de tórax: assegurar que sua fixação esteja adequada, não clampá-los, a não ser apenas para posicionamento do paciente na maca e transportar desclampado, com o frasco de drenagem num nível abaixo do ponto de inserção do dreno na parede torácica.

- Cateteres venosos: assegurar que a fixação esteja adequada, não deixá-los tracionados pelos equipos de soro e certificar-se de que os soros infundidos não irão acabar. 
É importante que haja a antecipação das posições dos equipos e frascos de soro para que não haja a tração e retirada acidental dos cateteres venosos periféricos ou centrais, principalmente, quando o paciente irá ser mudado de posição ou de maca. Tal fato causa desconfortos, além dos riscos e atrasos ocasionados por uma nova punção.

- Sondas nasogástrica e vesical: assegurar que a fixação esteja adequada. As sondas podem ser clampadas apenas por curtos períodos e, transportadas no mesmo nível do paciente.

Todos estes cuidados devem ser redobrados quando o paciente for transferido para outro leito, pois é no momento da passagem do paciente para outras macas, camas ou mesas, que ocorrem os maiores problemas.

Não é obrigatória a presença de equipamentos de reanimação cardiopulmonar e de sucção para acompanhar cada paciente que está sendo transportado, porém tais equipamentos devem estar localizados em áreas usadas por pacientes críticos e devem estar disponíveis dentro de, no máximo 4 minutos, para atender emergências que possam ocorrer no transporte.

Alguns serviços possuem uma unidade móvel de transporte permanentemente equipada, que é composta por um ventilador mecânico, monitor das funções vitais e bombas de infusão ${ }^{8,24}$.

\section{2- FASE DE TRANSFERÊNCIA}

O objetivo nesta fase é manter a estabilidade fisiológica do paciente através de monitorização contínua e prevenção para evitar iatrogenias.

O paciente que está sendo transportado deve receber a mesma monitorização das funções vitais, semelhante àquela que estava sendo oferecida na CTI, se isto for tecnologicamente possível.

O nível mínimo de monitorização para todo paciente em transporte deve ser: eletrocardiografia, frequiência cardíaca e respiratória e oximetria de pulso, de modo contínuo, e a medida intermitente da pressão arterial não invasiva ${ }^{20,21}$. Alguns pacientes selecionados podem se beneficiar da monitorização da capnografia, pressão arterial invasiva, pressão da artéria pulmonar e pressão intracraniana e medidas intermitentes da pressão venosa central, pressão capilar pulmonar e débito cardíaco.

Nos pacientes intubados que estão conectados a um ventilador mecânico, a pressão das vias aéreas deve ser monitorizada. Os alarmes do ventilador mecânico que indicam desconexão ou pressão excessivamente alta nas vias aéreas devem estar habilitados.

Esta é a fase mais neglicenciada dentre os transportes intra-hospitalares, pois os pacientes ficam fora da área de cuidados intensivos, em locais onde a monitorização é difícil e sem equipamento suficiente para controlar situações de emergência.

As intercorrências ocorrem, principalmente, por episódios relacionados aos equipamentos: deslocamento da cânula endotraqueal, perda do suprimento de oxigênio, mau funcionamento do equipamento por falta de energia, bateria descarregada ou defeito do aparelho, perda de cateter endovenoso ficando sem receber hidratação e medicações, retirada de sonda nasogástrica ou vesical. Aqui, principalmente, é notada a importância da fase preparatória do transporte, pois com a seleção e manutenção prévia dos materiais e equipamentos necessários, a grande maioria das intercorrências pode ser evitada.

Os cuidados devem ser redobrados quando é necessário transferir o paciente do seu leito para outro leito ou maca, com retorno ao leito original a seguir. É neste momento que costuma ocorrer a maior parte das intercorrências, como no transporte para a realização de tomografia computadorizada, que em alguns trabalhos podem representar até $71 \%$ das intercorrências ${ }^{7,30}$. Esta alta porcentagem de intercorrências também reflete o isolamento físico do paciente durante a realização do exame e a alta proporção da necessidade de permanência no local ${ }^{30}$.

\section{3- ESTABILIZAÇÃO PÓS-TRANSPORTE}

O paciente crítico pode apresentar-se com estabilidade hemodinâmica ao longo do transporte e vir a ter alterações hemodinâmicas apenas após o final de todo esse processo, ou seja, de volta ao CTI ou outras áreas de cuidados intensivos.

Após as alterações ocorridas ao longo do transporte, a estabilização do paciente crítico é lenta. Portanto, deve-se considerar um período de meia à uma hora após o transporte como uma fase de extensão do mesmo. Não é algo difícil de ser feito, pois o paciente estará de volta ao ambiente protegido do CTI.

Recomenda-se-maior atenção aos parâmetros hemodinâmicos e respiratórios nessa fase.

Muitos hospitais têm documentado a evolução do transporte intra-hospitalar, a fim de obter um melhor conhecimento de sua casuística, visando um melhor controle de qualidade. 
Pereira Júnior GA, Carvalho JB, Ponte Filho AD, Malzone DA, Pedersoli CE. Intra-hospital transport of critical patients. Medicina (Ribeirão Preto) 2007; 40 (4): 500-8, oct./dec.

ABSTRACT: The medicine knowledge and development of new technologies for diagnoses and treatment of patients had permitted the longevity of healthy people and of those with acute or chronic illness. These technological advances are not well distributed, but there are clearly improvements on the management of patients in medical field leading to a lower mortality and a better quality of life. With these improvements, hospitals needed to increase the area of critical care settings. Patients have being beneficiated by these diagnose technologies, most of them including image, but to access them, they need to be transported out of a intensive care unit, needing to maintain the same level of monitorization. That is the great importance on transportation of a critical patient, and it has being neglected by most health professionals. The objective of this article is to make a reflection of the various moments, phases and care involving the intra-hospital transport, discussing its various aspects.

Keywords: Transportation of Patient. Intensive Care. Monitoring. Patient Transfer.

\section{REFERÊNCIAS}

1 - Guidelines Committtee of the American College of Critical Care Medicine. Society of Critical Care Medicine and American Association of Critical Care Nurses Transfer Guidelines Task Force: Guidelines for the transfer of critically ill pacients. Crit Care Med 1993; 21:931-7.

2 - Manji M, Bion JF. Transporting critically ill patients. Intensive Care Med 1995; 21:781-3

3 - Braman SS, Dunn SM, Amico CA, Millman RP. Complications of intrahospital transport in critically ill patients. Ann Intern Med 1987; 107: 469-73.

4 - Smith I, Fleming S, Cernaiana A. Mishaps during transport from the intensive care unit. Crit Care Med 1990; 18:278-81.

5 - Insel J, Weissman C, Kemper M, Askanazi J, Hyman AL. Cardiovascular changes during transport of critically ill and postoperative patients. Crit Care Med 1986; 14:539-42.

6 - Ehrenwerth J, Sorbo S, Hackel A. Transport of critically ill adults. Crit Care Med 1986; 14:543-7.

7 - Link J;,Krause H, Wagner W, Papadopoulos G. Intrahospital transport of critically ill patients. Crit Care Med 1990; 18: 1427-9.

8 - Waydhas C, Scheneck G, Duswald KH. Deterioration of respiratory function after intra-hospital transport of critically ill surgical patients. Intensive Care Med 1995; 21:784-9.

9 - Indeck M, Peterson S, Smith J, Brotman S. Risk, cost and benefit of transporting ICU patients for special studies. J Trauma 1988; 28:1020-5.

10 - Hurst JM, Davis K JR, Johnson D, Branson RD, Campbell RS, Branson PS. Cost and complications during in-hospital transport of critically ill patients: A prospective cohort study. J Trauma 1992; 33:582-5.
11 - Nakamura T, Fujino Y, Uchiyama A, Mashimo T, Nishumura M. Intrahospital transport of critically ill patients using ventilador with patient-triggering function. Chest 2003; 123: 159-64.

12 - Knaus WA, Draper EA, Wagner D, Zimmerman JE. APACHE II: A severity of disease classification. Crit Care Med 1985; 13:818-29.

13 - Bion JF, Wilson IH, Taylor PA. Transferring critically ill patients by ambulance: audit by sickness scoring. Br Med J 1988; 296:170-5.

14 - Bion JF, Edlin AS, Ramsay G, McCabe S, Ledingham IM. Validation of a prognostic score in critically ill patients undergoing transport. Br Med J 1985; 291:432-4.

15 - Kanter R, Tompkins J. Adverse events during interhospital transport: physiologic deterioration associated with pretransport severity of illness. Pediatrics 1989; 84:43-8.

16 - 16 - Orr RA, Venkataraman ST, Cinoman MI, Hogue BL, Singleton CA. Pretransport pediatric risk of mortality (PRISM) score underestimates the requirement for intensive care or major interventions during interhospital transport. Crit Care Med 1994; 22:101-7.

17 - Szem JW, Hydo LJ, Fisher E, Kapur S, Klemperer J, Barie PS. High-risk intrahospital transport of critically ill patients: safety and outcome of the necessary "road trip". Crit Care Med 1995; 23: 1660-6.

18 - Lee SY, Frankel HL. Ultrasound and other imaging technologies in the intensive care unit. Surg Clin Nort Am 2000; 80: 975-1003.

19 - McCunn M, Mirvis S Reynolds N, Cottingham C. Physician utilization of a portable computed tomography scanner in the intensive care unit. Crit Care Med 2000; 28: 3808-13.

20 - Warren J, Fromm RE Jr, Orr RA, Rotello LC, Horst HM. Guidelines for the inter- and intrahospital transport of critically ill patients. Crit Care Med 2004; 32: 256-62. 
21 - Australasian College for Emergency Medicine, Australian and New Zealand College of Anaesthetists, Joint Faculty of Intensive Care Medicine. Minimum standards for intrahospital transport of critically ill patients. Emerg Med 2003; 15: 2024.

22 - AARC Clinical Practice Guideline. In-hospital transport of the mechanically ventilated patient. Respir Care 2002; 47: 7213.

23 - Weg JG, Haas CF. Safe intrahospital transport of critically ill ventilator-dependent patients. Chest 1989; 96:631-5.

24 - Barton ACH, Tuttle-Newhall JE, Szalados JE. Portable power supply for continuous mechanical ventilation during intrahospital transport of critically ill patients with ARDS. Chest 1997; 112: 560-3.

25 - Hackel A, Simon JE, Wingert WA. Committee on hospital care guidelines for air and ground transportation of pediatric patients. Pediatrics 1986; 78: 943-50.

26 - Day S, McCloskey K. Pediatric interhospital critical care transport: Consensus of national leadership conference. Pediatrics 1991; 88: 696-704.
27 - Macdonald MG. Infant transport equipment checklist. In: MacDonald MG, Miller MK, eds. Emergency transport of the perinatal patient. Boston: Little, Brown;1989. p. 410-22.

28 - Giles HR. Maternal transport. Clin Obstet Gynecol 1979; 6: 203-14.

29 - Andrews P, Piper I, Dearden NM, Miller JD. Secondary insults during intrahospital transport of head-injured patients. Lancet 1990; 335(8685):327-330.

30 - Conn AKT. Transport of the critically ill patient. In: Shoemaker, Ayres, Grenvik, Holbrook. Textbook of critical care. 3th ed. Philadelphia, Pennsylvania: W.B. Saunders; 1995: 233-254.

Recebido em 29/06/2007

Aprovado em 31/01/2008 